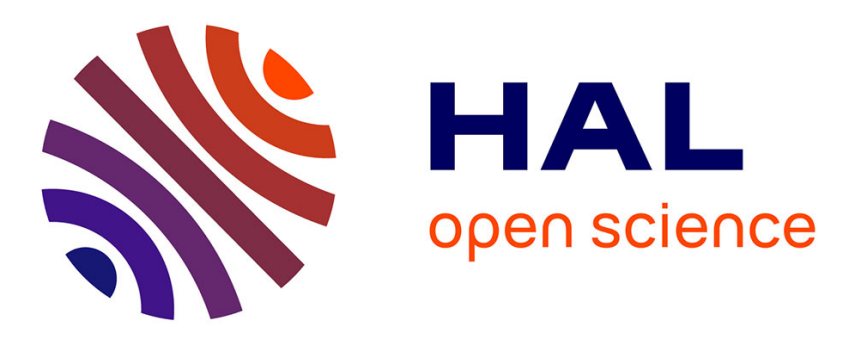

\title{
Effective field and universal mobility in high-k metal gate UTBB-FDSOI devices
}

\author{
Olivier Nier, Denis Rideau, Antoine Cros, Frédéric Monsieur, Gérard \\ Ghibaudo, Raphael Clerc, Jean-Charles Barbé, Clément Tavernier, Hervé \\ Jaouen
}

\section{To cite this version:}

Olivier Nier, Denis Rideau, Antoine Cros, Frédéric Monsieur, Gérard Ghibaudo, et al.. Effective field and universal mobility in high-k metal gate UTBB-FDSOI devices. 27th International Conference on Microelectronic Test Structures (ICMTS), Mar 2014, Udine, Italy. pp.8 - 13, 10.1109/ICMTS.2014.6841460 . hal-01102474

\section{HAL Id: hal-01102474 \\ https://hal.science/hal-01102474}

Submitted on 13 Jan 2015

HAL is a multi-disciplinary open access archive for the deposit and dissemination of scientific research documents, whether they are published or not. The documents may come from teaching and research institutions in France or abroad, or from public or private research centers.
L'archive ouverte pluridisciplinaire HAL, est destinée au dépôt et à la diffusion de documents scientifiques de niveau recherche, publiés ou non, émanant des établissements d'enseignement et de recherche français ou étrangers, des laboratoires publics ou privés. 


\title{
Effective field and universal mobility in high-k metal gate UTBB- FDSOI devices.
}

\author{
O. Nier ${ }^{1,2,3,4}$, D. Rideau ${ }^{1}$, A. Cros ${ }^{1}$, F.Monsieur $^{1}$, G. Ghibaudo ${ }^{2}$, R. Clerc ${ }^{5}$, J.C Barbé ${ }^{3}$, C. Tavernier ${ }^{1}$, H. Jaouen $^{1 .}$
} 1) STMicroelectronics, 850, rue J. Monnet, BP. 16, 38921 Crolles, France ; 2) IMEP-LAHC, MINATEC 3 Parvis Louis Néel, 38016 Grenoble ;

3) CEA-LETI, Campus MINATEC, 17 rue des Martyrs, 38054 Grenoble, France ; 4) DIEGM, University of Udine, Via delle Scienze 208, 33100 Udine, Italy; 5) Laboratoire Hubert Curien (UMR 5516), Institut d'Optique Graduate School, 18 rue Benoît Lauras 42000, Saint-Etienne, France.

\begin{abstract}
This paper aims at reviewing experimental and theoretical behaviors of universal mobility in high-k metal gate UTBB-FDSOI devices. Based on split-CV mobility measurements, the parameter $\eta$, characterizing the effective field, has been extracted for a large range of back voltages and temperatures in devices with various equivalent oxide thicknesses. We demonstrated that a nearly universal trend for the mobility with respect to the effective field can be obtained in the front inversion regime but is difficult to obtain in the back channel inversion regime.
\end{abstract}

Keywords-FDSOI, universal mobility, effective field, coefficient $\eta$.

\section{INTRODUCTION}

Ultra-Thin Body and Box Fully Depleted SOI (UTBB-FDSOI) device is considered as one of the best alternative solution to overcome conventional planar Bulk CMOS limitations [1-2]. Transport properties in UTBB-FDSOI have been extensively studied and many issues have been reported concerning both, the definition of the effective field $\left(E_{\text {eff }}\right)[3]$ and the universal behavior of the mobility [4]. This paper aims at reviewing important experimental and theoretical aspects of the universal behavior of the mobility with respect to the effective field. The parameter $\eta$, characterizing the effective field, has been extracted from split-CV mobility measurements for $\mathrm{n}$ - and $\mathrm{p}$ type UTBB-FDSOI devices. These extractions have been performed for a large range of Back gate Voltages (VB), temperatures and for devices with various Interfacial oxide (IL) Equivalent Oxide Thicknesses (EOT).

\section{EXPERIMENTAL OBSERVATIONS}

Devices have been processed on $300 \mathrm{~mm}$ SOI wafers with a 25 $\mathrm{nm}$ thick BOX. The final channel thickness is $7.5 \mathrm{~nm}$ and the oxide stack is made of a $1.8 \mathrm{~nm}$ thick layer of HfSiON high-k oxide deposited on top of a SiON interfacial layer (IL) with various physical thicknesses ( $\mathrm{T}_{\text {inv }}$ ranging from 1.3 to $4.0 \mathrm{~nm}$ for both $n$ - and p- FDSOI devices). The effective mobility has been characterized using split-CV method on $0.9 \times 0.9 \mu \mathrm{m}^{2}$ devices (measurements at $\mathrm{V}_{\mathrm{DS}}=50 \mathrm{mV}$ ) for a large range of $\mathrm{VB}$ $(-10 \mathrm{~V}<\mathrm{VB}<10 \mathrm{~V})$ and temperatures $(\mathrm{T}=233 \mathrm{~K}, 300 \mathrm{~K}$ and 450 $\mathrm{K})$. The impact of the $\mathrm{VB}$ on the capacitance and the drain current is shown in figure 1 for various temperatures. Figures 2 and 3 demonstrate a non monotonic behavior and an improvement of the mobility in the forward regime $(\mathrm{VB}>0 \mathrm{~V}$ for $\mathrm{nFDSOI}$ and $\mathrm{VB}<0 \mathrm{~V}$ for pFDSOI devices) which is a direct consequence of the back gate inversion, as previously observed in [5-6]. The extractions have been corrected from access resistances of $\mathrm{R}_{\mathrm{access}}=2 \times 60 \Omega . \mu \mathrm{m}$. This resistance has been extracted using the $\mathrm{R}_{\mathrm{on}}(\mathrm{L})$ method [7], i.e. from the intersect of the curve $\mathrm{Vds} / \mathrm{Ion}$ vs $\mathrm{L}$ at $\mathrm{L}=0$. In figure 2 , the mobility has been plotted as a function of the inversion charge for two different values of $\mathrm{R}_{\text {access }}$ indicating that the effect on mobility is more pronounced in the back inversion regime and emphasing the importance of an accurate deembedding of $\mathrm{R}_{\text {access. }}$.

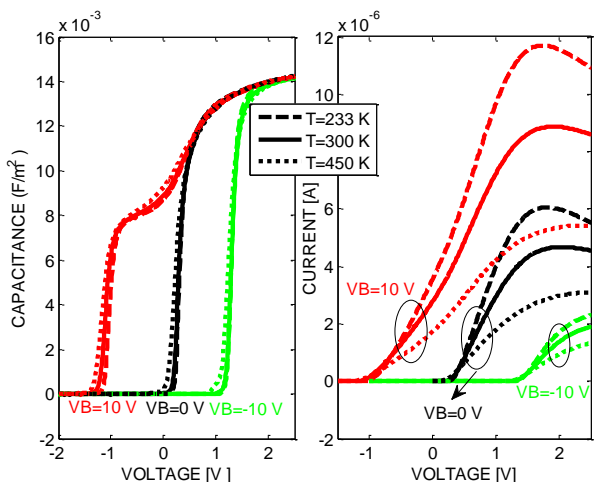

Figure 1: Gate capacitance (left) and drain current (right) as a function of the gate voltage for $V B=-10,0$ and $10 \mathrm{~V}$ and for various temperatures $(T=233$, 300 and $450 \mathrm{~K}$ ) in a $\mathrm{nFDSOI}$ device: $T_{i n v}=2.65 \mathrm{~nm}$.

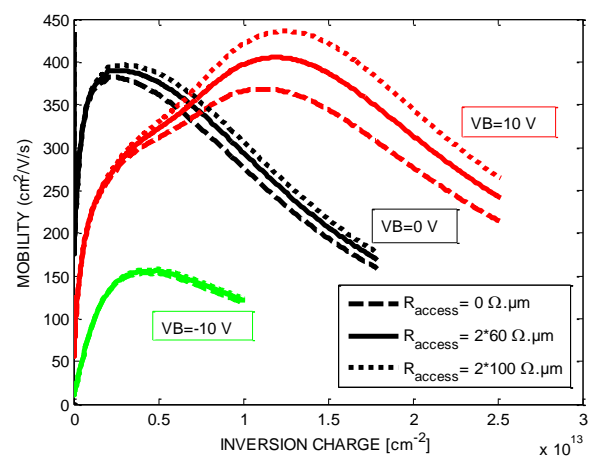

Figure 2: Effective experimental mobility as a function of the inversion charge for various $V B(-10 \mathrm{~V}, 0 \mathrm{~V}, 10 \mathrm{~V})$ corrected from access resistances (not corrected, $2 \times 60 \Omega . \mu m$ and $2 * 100 \Omega . \mu \mathrm{m}) . n F D S O I$ device $: T_{i n v}=2.65 \mathrm{~nm}$. $T=300 \mathrm{~K}$.

The variation of the capacitance and the threshold voltage (VTH) for all devices are shown in figures 4 and 5 respectively. It demonstrates that the VTH can be shifted by -.5 $\mathrm{V}$ to $1 \mathrm{~V}$ for $\mathrm{VB}$ ranging from $-10 \mathrm{~V}$ to $10 \mathrm{~V}$. The mobility degradation observed in figure 6 in the thinner EOT device is attributed to remote coulomb scattering due to the presence of charges at high-k/IL interface [6]. 

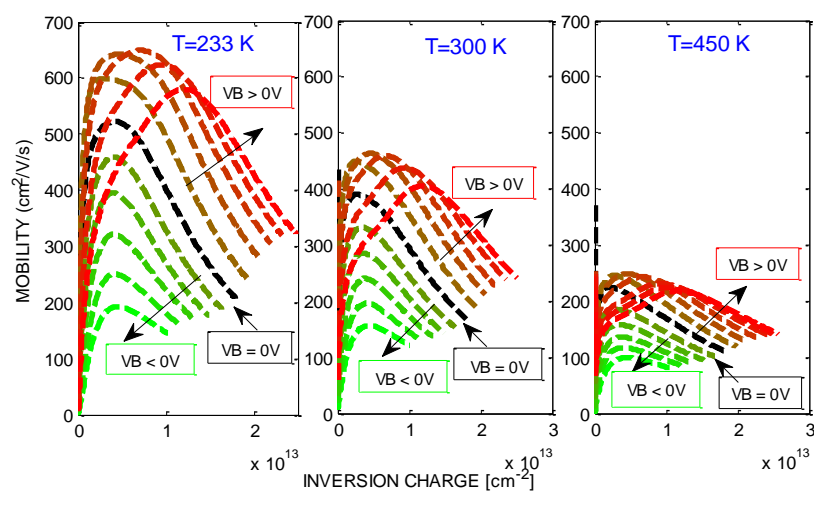

Figure 3: Effective experimental mobility as a function of the inversion charge for various $V B$ ranging from $-10 \mathrm{~V}$ to $10 \mathrm{~V}$ in steps of $2 \mathrm{~V}$. $n$ FDSOI device: $T_{i n v}=2.65 \mathrm{~nm} . T=233,300$ and $450 \mathrm{~K}$.

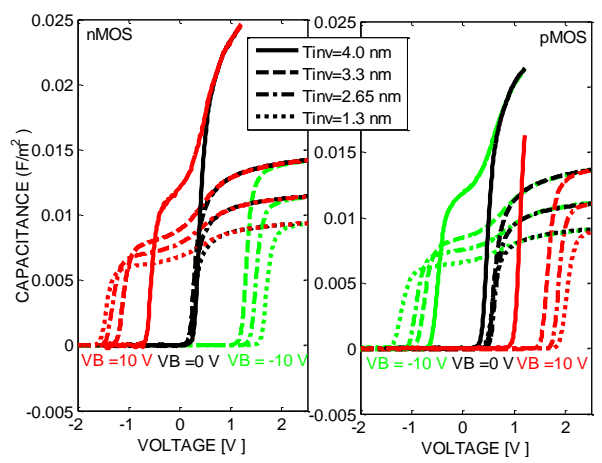

Figure 4: Gate capacitance as a function of the gate voltage for $V B=-10,0$ and $10 \mathrm{~V}$ in $\mathrm{n}$ - (left) and p- (right) FDSOI devices with $T_{\text {inv }}=1.3 \mathrm{~nm}, 2.65 \mathrm{~nm}$, $3.3 \mathrm{~nm}$ and $4.0 \mathrm{~nm} . \mathrm{T}=300 \mathrm{~K}$.

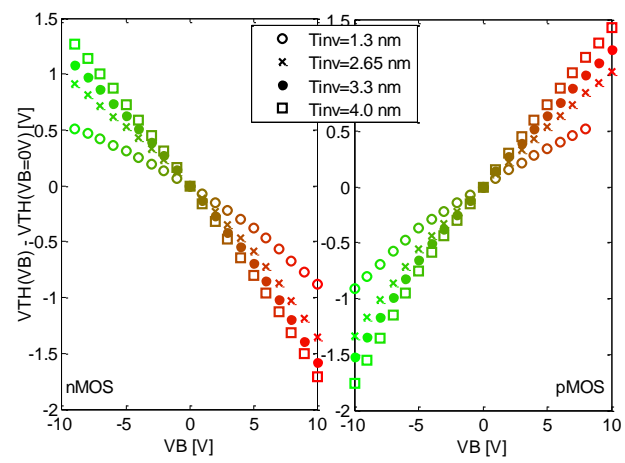

Figure 5: Threshold voltage variation (reference at $V B=0 \mathrm{~V}$ ) as a function of VB in n-(left) and p-(right) FDSOI devices. Devices of figure $4 . T=300 \mathrm{~K}$.

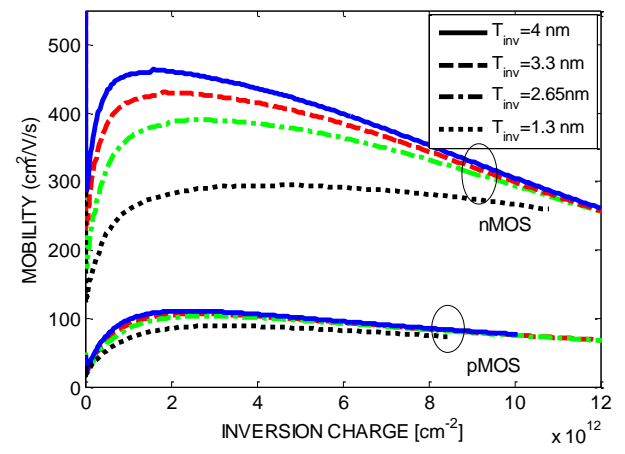

Figure 6: $n$ - and p-FDSOI experimental effective mobility as a function of the inversion charge. Devices of figure $4 . T=300 \mathrm{~K} ; \mathrm{VB}=0 \mathrm{~V}$.
The mobility extracted at fixed inversion charge as a function of VB (figure 7) exhibits a bell-shaped behavior [6]. In the next section, we show that this behavior can be further highlighted representing the mobility versus the effective field rather than as a function of the inversion density as in figure 2 .

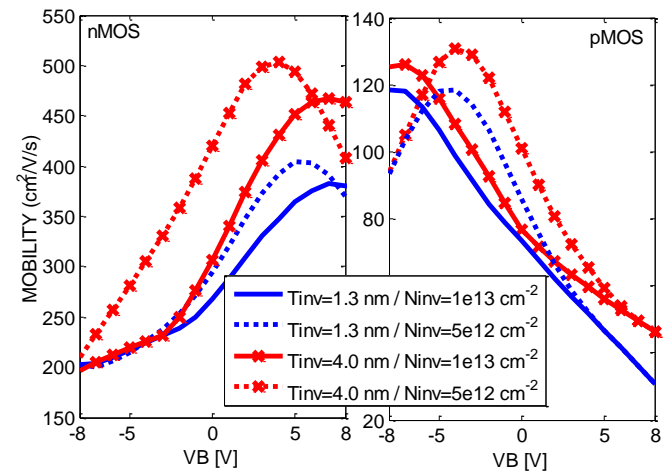

Figure 7: $n$ - (left) and p- (right) FDSOI experimental effective mobility as a function of $V B$ extracted for Ninv $=5 e 12 \mathrm{~cm}^{-2}$ and $1 e 13 \mathrm{~cm}^{-2}$. Two structures:

$$
T_{i n v}=1.3 \mathrm{~nm} \text { and } 4.0 \mathrm{~nm} . \mathrm{T}=300 \mathrm{~K} \text {. }
$$

\section{DEFINITION OF THE EFFECTIVE FIELD IN FDSOI TRANSISTORS AND EXTRACTION OF $\eta$ PARAMETER.}

A transverse average electric field can be defined from the local concentration $\mathrm{n}(\mathrm{x})$ and the transverse field $\mathrm{E}_{\mathrm{z}}(\mathrm{x})$ as:

$$
E_{\text {ave }}^{\text {th }}=\frac{\int_{0}^{T} S I E_{z}(x) n(x) d x}{\int_{0}^{T} S I n(x) d x}
$$

This later equation can be approximated by the following simplified equation in UTBB-FDSOI MOSFETs [8]:

$$
E_{\text {ave } / \text { eff }}^{\text {exp }}=\frac{Q_{d}+\eta Q_{i}}{\epsilon_{S i}}-\frac{\epsilon_{\text {ox }}}{\epsilon_{S i}} \frac{V B}{T_{B O X}^{e f f}}
$$

with $T_{B O X}^{e f f}=\frac{C_{S i}+C_{B O X}}{C_{S i}} T_{B O X}+\Delta, Q_{i}$ the inversion charge, $Q_{d}$ the depletion charge $\left(Q_{d}=q N_{a} T_{S I}\right)$ and $\Delta \simeq 1.5 \mathrm{~nm}$ accounts for the ground plane accumulation or depletion. In order to match equations (1) and (2), $\eta$ has to be taken equal to $\eta=0.5$ for both $n$ - and pMOS devices in bulk [9] as well as in FDSOI structures [10].

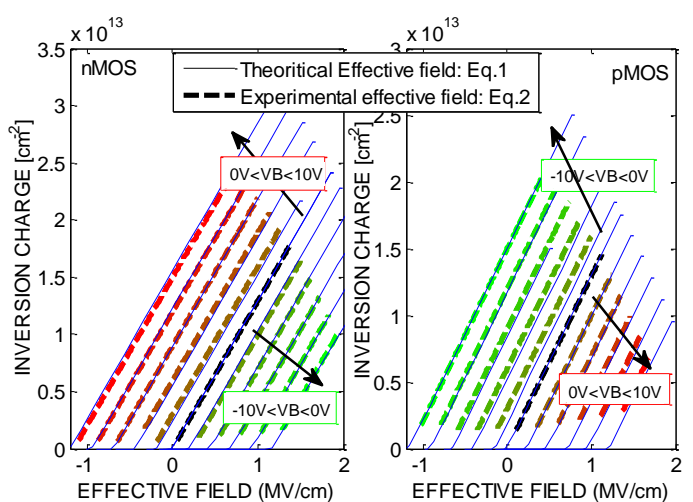

Figure 8: $n$ - (left) and p- (right) FDSOI average electric field (Eq.2) assuming $\eta=0.5$ compared to the simulated theoretical average field (Eq.1). VB from -

$10 \mathrm{~V}$ to $10 \mathrm{~V}$ in steps of $2 \mathrm{~V}$. $T_{\text {inv }}=2.65 \mathrm{~nm} ; \mathrm{T}=300 \mathrm{~K}$.

Figure 8 demonstrates that the approximated average field from Eq. 2 reproduces accurately the numerical one (Eq.1) calculated using a self-consistent Poisson-Schrödinger solver for all biases 
conditions. This also provides an efficient way to calibrate parameters $\mathrm{T}_{\mathrm{si}}, \varepsilon_{\mathrm{ox}}$, IL EOT, $\mathrm{T}_{\mathrm{BOX}}$ from experiments.

Another effective field $\mathrm{E}_{\text {eff }}$ (not to be confused with the average transverse average electric field) based on Eq.2 is usually used to get the universal mobility curve [11]. In this case, $\eta$ is used as an empirical parameter and is extracted from experiments. In the case of bulk devices, this parameter has been found equal to $\eta=0.5$ for bulk nMOSFET and $\eta=0.33$ for bulk pMOSFET [11].

In high-k UTBB-FDSOI ( $\mathrm{T}_{\mathrm{inv}}$ ranging from 1.3 to $4 \mathrm{~nm}$ ), it has been demonstrated $[5,6]$ that the main mechanisms responsible of the mobility degradation are remote coulomb (due to the presence of charges at high-k/IL interface), phonons and surface roughness scatterings. From theoretical mobility calculation, we have shown in a previous work [5] that, in the back inversion regime or for devices with thick EOT $\left(\mathrm{T}_{\mathrm{IL}}>2.0\right.$ $\mathrm{nm}$ ), the remote coulomb component does not play a significant role in the total mobility. It is thus interesting to study the impact of remote coulomb scattering on mobility curves changing VB, temperatures or IL EOT.

Using values extracted in bulk devices, the experimental mobility has been plotted in the case of FDSOI devices as a function of the effective field for various VB ranging from -10 $\mathrm{V}$ to $10 \mathrm{~V}$ (figure 9). First of all, it can be noticed that the effective field spans a much larger range of values when sweeping VB. However, it is clear from this figure that the mobility does not follow a universal trend, in particular in the back inversion regime.

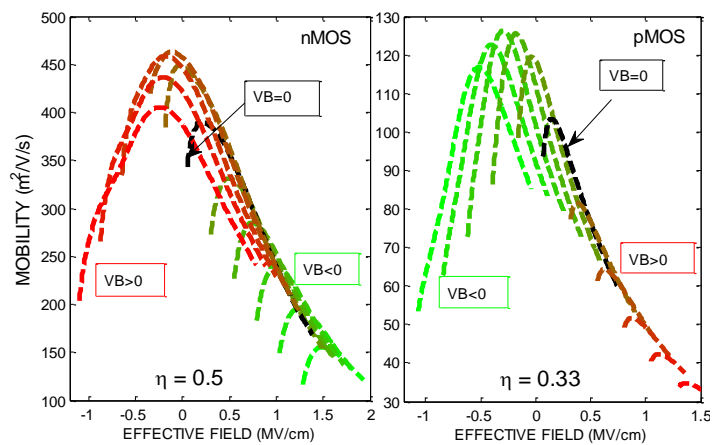

Figure 9: $n$ - (left) and p- (right) FDSOI experimental effective mobility as a function of the effective field. $\mathrm{VB}$ ranging from $-10 \mathrm{~V}$ to $10 \mathrm{~V}$ in steps of $2 \mathrm{~V}$. Effective field calculated using Eq.2 ( $\eta=0.5$ for $n F D S O I$ and $\eta=0.33$ for pFDSOI). $T_{i n v}=2.65 \mathrm{~nm} . T=300 \mathrm{~K}$.

A recent paper has shown that the value of $\eta$ in FDSOI devices may be different from bulk values [12]. This raises the question of the extraction of this parameter. $\eta$ is usually extracted by adjusting experimental curves in order to get the best universal trend. However, an alternative technique has been recently proposed, deducing $\eta$ from the following formula [13]:

$$
\eta_{\text {SiOI }}=\frac{A C_{b o x}}{A C_{B}-C_{o x}} \text { with } A=\frac{\left.\left(\partial \mu_{e f f} / \partial V_{f g}\right)\right|_{V_{b g}}}{\left.\left(\partial \mu_{e f f} / \partial V_{b g}\right)\right|_{V_{f g}}}
$$

This method (Eq.3) has been used to extract $\eta$ at $\mathrm{VB}=0 \mathrm{~V}$ for various temperatures (figure 10), leading to $\eta=0.42$ and 0.36 for $n$ - and p- FDSOI devices respectively. A slight decrease of the coefficients has been observed for increasing temperatures.
This can also be seen in figure 11 where $\eta$ has been characterized for devices with various IL EOT.

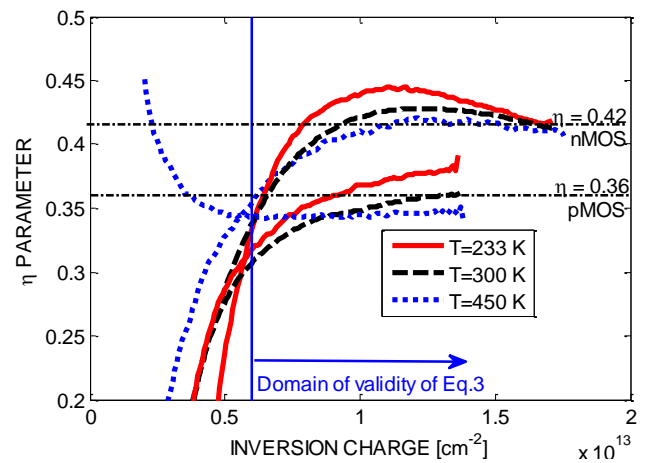

Figure 10: Extracted value of parameter $\eta$ using Eq. 3 in $n$ - and pFDSOI devices for various temperatures $(T=233,300$ and $450 \mathrm{~K})$. $n F D S O I$ device :

$T_{i n v}=2.65 \mathrm{~nm}$. The domain of validity corresponds to the strong front inversion regime.

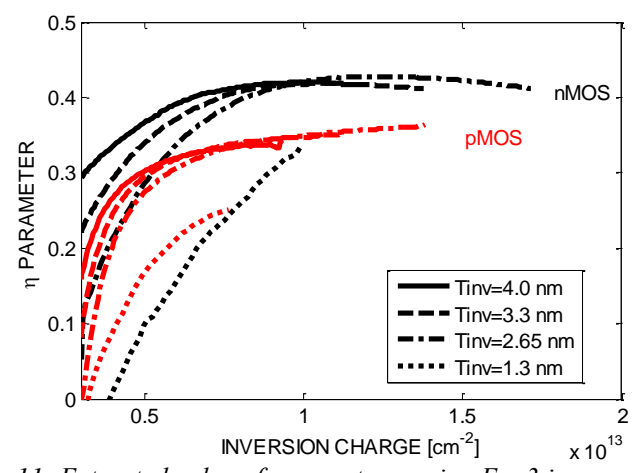

Figure 11: Extracted value of parameter $\eta$ using Eq. 3 in $n$ - and pFDSOI devices with various $T_{i n v}$ (devices of figure 4 ). $T=300 \mathrm{~K} . \mathrm{VB}=0 \mathrm{~V}$.

Using these extracted values $\eta=0.42$ and 0.36 , the experimental mobility has been plotted as a function of the effective field (figure 12). Also in this case, it turns out that the mobility does not follow a universal trend.

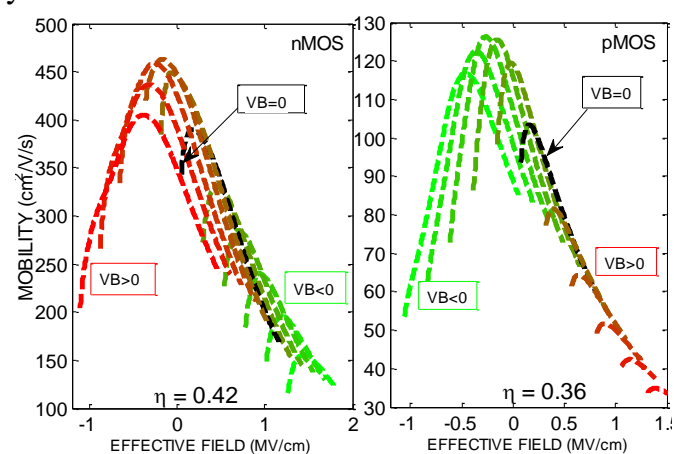

Figure 12: $n$ - (left) and p- (right) FDSOI experimental effective mobility as a function of the effective field. VB ranging from $-10 \mathrm{~V}$ to $10 \mathrm{~V}$ in steps of $2 \mathrm{~V}$. Effective field calculated using Eq.2 ( $\eta=0.42$ for $n F D S O I$ and $\eta=0.36$ for pFDSOI). $T_{i n v}=2.65 \mathrm{~nm} . \mathrm{T}=300 \mathrm{~K}$.

It ought to be notified that Eq.3 is valid only when the front interface is in the strong inversion regime [13]. However it can be interesting to apply this equation with reverse $\mathrm{VB}(\mathrm{VB}>0 \mathrm{~V}$ for $\mathrm{nFDSOI}$ and $\mathrm{VB}<0 \mathrm{~V}$ for pFDSOI devices) since at high gate voltages, the front channel is also inverted. In figures 13 and $14, \eta$ has been extracted for $\mathrm{VB}$ varying from $-10 \mathrm{~V}$ to 10 $\mathrm{V}$ in $\mathrm{n}$ - and $\mathrm{p}$ - FDSOI devices. In the front inversion regime, $\eta$ 
converge to the previously extracted values while in the back inversion regime, an increase of the coefficients with VB can be observed. As expected when the front channel is not inverted, Eq. 3 is not valid and the extraction of $\eta$ is not relevant.

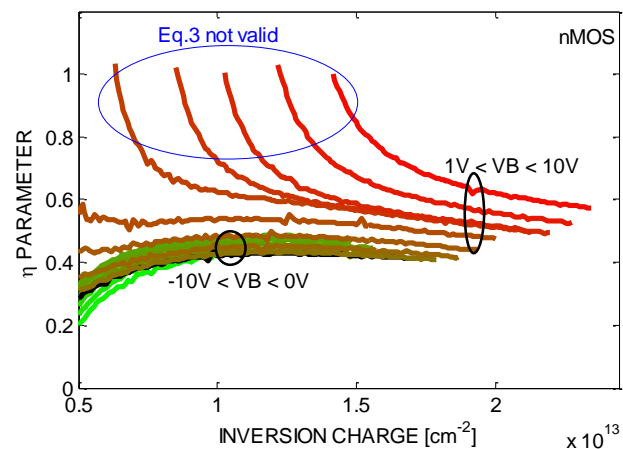

Figure 13: Extracted value of parameter $\eta$ using Eq. 3 in a $\mathrm{nFDSOI}$ device for various $V B$ ranging from $-10 \mathrm{~V}$ to $10 \mathrm{~V}$. $T_{i n v}=2.65 \mathrm{~nm} . T=300 \mathrm{~K}$. In the front inversion regime $(V B<0), \eta \simeq 0.42$ while in the forward regime $(V B>0), \eta$ varies significantly with $V B$.

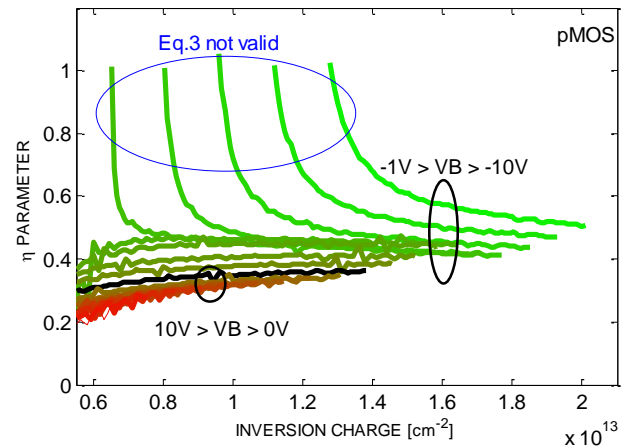

Figure 14: Extracted value of parameter $\eta$ using Eq.3 in a pFDSOI device for various $\mathrm{VB}$ ranging from $-10 \mathrm{~V}$ to $10 \mathrm{~V}$. $T_{\text {inv }}=2.65 \mathrm{~nm} . T=300 \mathrm{~K}$. In the front inversion regime $(V B>0), \eta \simeq 0.36$ while in the forward regime $(V B<0), \eta$ varies significantly with $V B$.

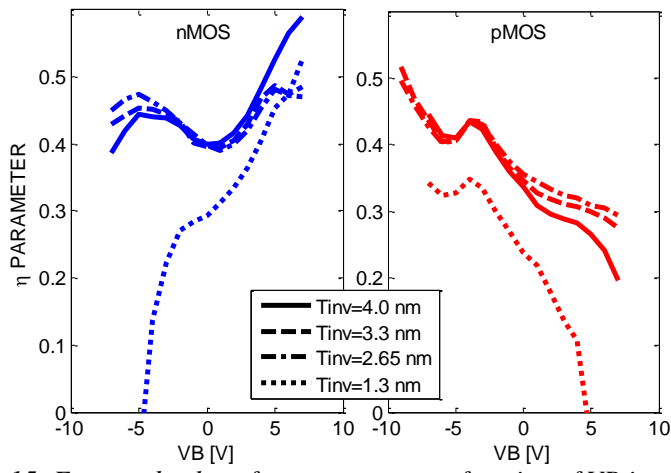

Figure 15: Extracted value of parameter $\eta$ as a function of $V B$ in $n$ - and $p$ FDSOI devices with various IL EOT (devices of figure 4). $\eta$ extracted using Eq.3. $T=300 \mathrm{~K}$. Extractions have been done at $V G=2.5 \mathrm{~V}$ for devices with $T_{i n v}=2.65,3.3$ and $4.0 \mathrm{~nm}$ and at $V G=1.2 \mathrm{~V}$ for devices with $T_{i n v}=1.3 \mathrm{~nm}$.

In figure 15, extracted values of parameter $\eta$ have been represented as a function of VB for $\mathrm{n}$ - and $\mathrm{p}$ - FDSOI devices with various IL EOT (devices of figure 4). It can be noticed that the three thicker IL EOT devices have roughly similar values for $\eta$ while in the thinnest devices, where the remote coulomb scattering plays a significant role, lower values can be observed.
In figure $16, \eta$ has been extracted for $n-$ and pFDSOI devices $\left(\mathrm{T}_{\mathrm{inv}}=4.0 \mathrm{~nm}\right)$ at various temperatures. As previously observed in figure 10 , the coefficients is not affected significantly by the temperature.

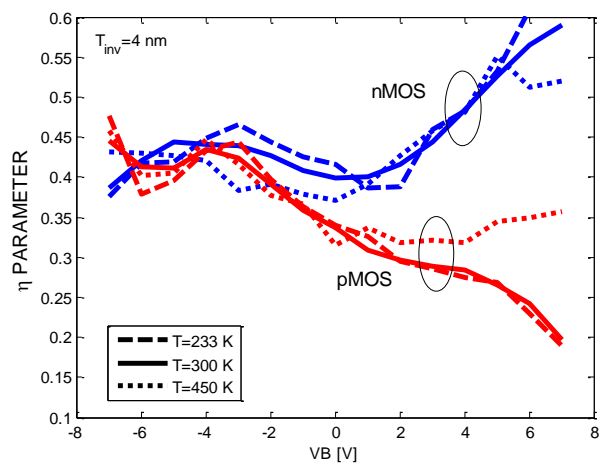

Figure 16: Extracted value of parameter $\eta$ as a function of $V B$ in $n$ - and pFDSOI devices for three different temperatures $(T=233,300$ and $450 \mathrm{~K}) . \eta$ extracted using Eq.3. $T_{i n v}=4.0 \mathrm{~nm}$. Extractions have been done at $V G=2.5 \mathrm{~V}$.

There is another aspect to mention concerning the key assumption behind Eq.3. It ought to be notified that this equation is based on the assumption that [13]:

$$
\left.\left(\partial \mu_{e f f} / \partial E_{e f f}\right)\right|_{V_{b g}}=\left.\left(\partial \mu_{e f f} / \partial E_{e f f}\right)\right|_{V_{f g}}
$$

In order to check the validity of this formula, in figures 17,18 and 19, the mobility has been plotted with respect to the effective field sweeping VB at fixed VG and sweeping VG at $\mathrm{VB}=0 \mathrm{~V}$. First of all, in figure 17, bulk values for $\eta$ are used ( 0.5 for $n-$ and 0.33 for pFDSOI devices) and the condition described in Eq.4 is not satisfied at high Eeff. However, the same conclusions can be obtained from figure 18 when using values extracted from Eq.3 $(\eta=0.42$ and 0.36 for $n-$ and pFDSOI devices respectively). In order to get a better agreement, $\eta$ also has been re-extracted by brute-force numerical fitting, leading to the value of $\eta=0.62$ for nFDSOI and $\eta=0.5$ for $p F D S O I$ devices. Results are shown in figure 19 . It can be notified incidentally that the behaviors shown in figure 17, 18 and 19 have been reproduced using a KuboGreenwood solvers (not shown here) [5].

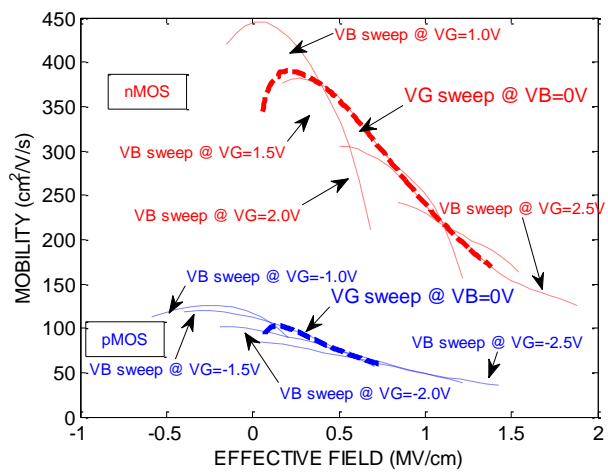

Figure 17: $n$ - and p- FDSOI experimental effective mobility as a function of the effective field sweeping the back terminal or the gate terminal. Eq.2 have been used for the evaluation of the effective field with $\eta=0.5$ for $n F D S O I$ and $\eta=0.33$ for $p F D S O I$ devices. $T_{i n v}=2.65 \mathrm{~nm} . T=300 \mathrm{~K}$. 


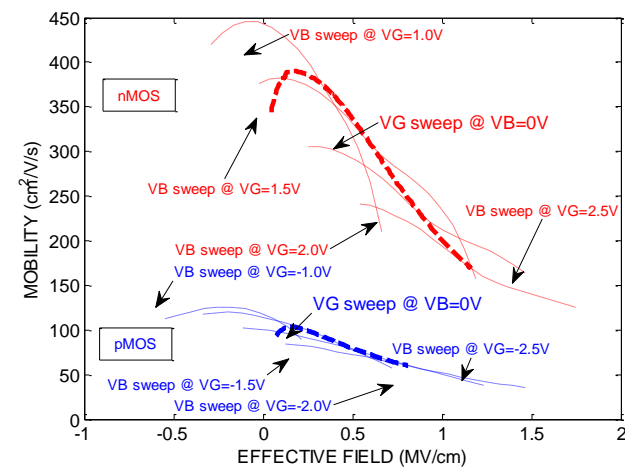

Figure 18: $n$ - and p- FDSOI experimental effective mobility as a function of the effective field sweeping the back terminal or the gate terminal. Eq.2 have been used for the evaluation of the effective field with $\eta=0.42$ for $n F D S O I$ and $\eta=0.36$ for pFDSOI devices. $T_{\text {inv }}=2.65 \mathrm{~nm} . T=300 \mathrm{~K}$.

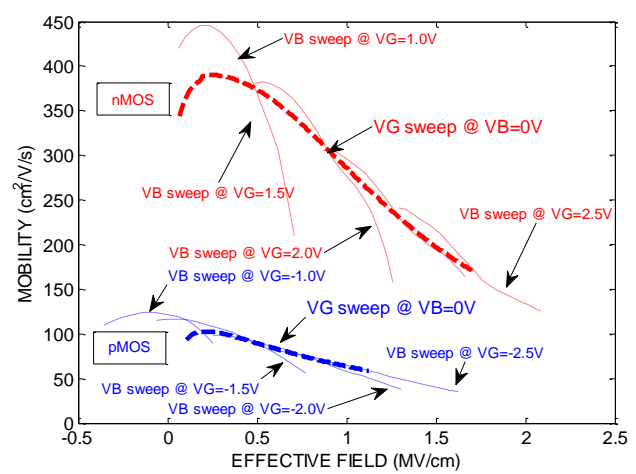

Figure 19: $n$ - and p- FDSOI experimental effective mobility as a function of the effective field sweeping the back terminal or the gate terminal. Eq.2 have been used for the evaluation of the effective field with $\eta=0.62$ for $n F D S O I$ and $\eta=0.5$ for pFDSOI devices. $T_{i n v}=2.65 \mathrm{~nm} . T=300 \mathrm{~K}$.

The variation of the mobility with respect to the effective field has been plotted for $\mathrm{VB}$ ranging from $-10 \mathrm{~V}$ to $10 \mathrm{~V}$ in figure 20 ( $\mathrm{n}$ - and p- FDSOI devices). In these cases, a much better universal trend is observed, although not perfect, in particular in the forward regime, as expected from the results shown in figures 13 and 14.
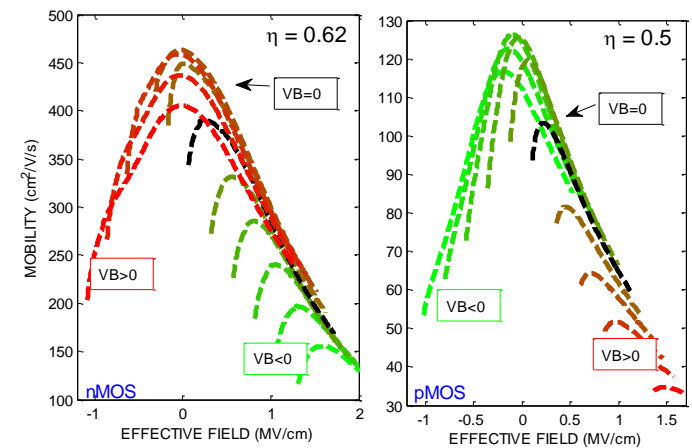

Figure 20: $n$ - (left) and p- (right) FDSOI experimental effective mobility as a function of the effective field. $\mathrm{VB}$ ranging from $-10 \mathrm{~V}$ to $10 \mathrm{~V}$ in steps of $2 \mathrm{~V}$. Effective field calculated using Eq.2 ( $\eta=0.62$ for $n F D S O I$ and $\eta=0.5$ for pFDSOI). $T_{i n v}=2.65 \mathrm{~nm} . \mathrm{T}=300 \mathrm{~K}$.

The mobility has been plotted as a function of effective field for n- (figure 21) and p- FDSOI (figure 22) devices with
Tinv=4.0 and $1.3 \mathrm{~nm}$. We can notice that for the thinnest device $($ Tinv $=1.3 \mathrm{~nm})$, where remote coulomb scattering plays a significant role, the mobility curves with respect to Eeff does not follow an unique trend even in the front inversion regime. The impact of remote coulomb scattering is further depicted in figure 23 where the mobility has been plotted for $\mathrm{VB}=-8 \mathrm{~V}, 0 \mathrm{~V}$ and $8 \mathrm{~V}$ for the nFDSOI devices with various IL EOT. This is also noticeable in pFDSOI devices where the remote coulomb scattering contribution to the total mobility is less pronounced.
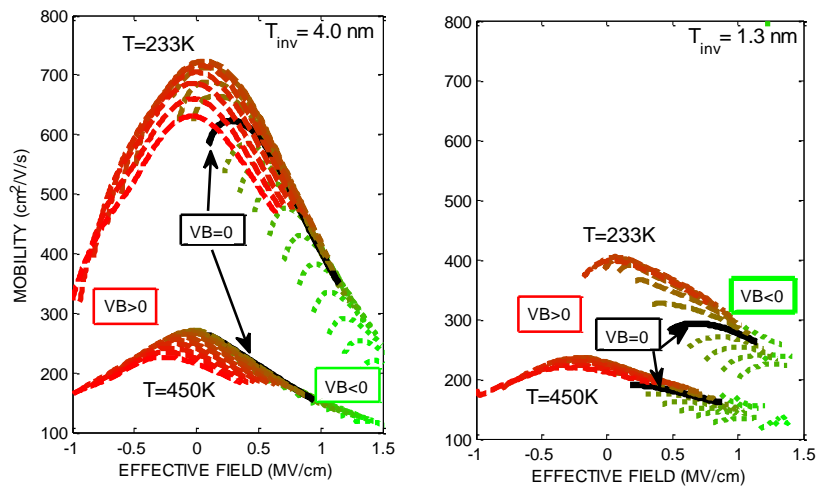

Figure 21: nFDSOI experimental effective mobility as a function of the effective field for $V B$ in devices with $T_{i n v}=4.0 \mathrm{~nm}$ (left) and $T_{i n v}=1.3 \mathrm{~nm}$ (right). Effective field calculated using Eq.2 $(\eta=0.62) . T=233 \mathrm{~K}$ and $450 \mathrm{~K}$. Measurements have been performed at zero $V B$ (line), but also for $V B>0$ (dashed lines) and $V B<0$ (dotted lines).
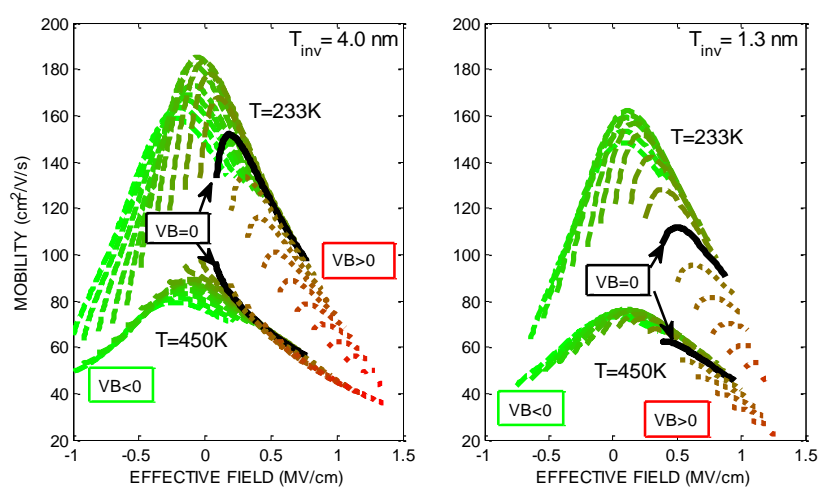

Figure 22: pFDSOI experimental effective mobility as a function of the effective field in devices with $T_{i n v}=4.0 \mathrm{~nm}$ (left) and $T_{i n v}=1.3 \mathrm{~nm}$ (right). Effective field calculated using Eq.2 ( $\eta=0.62) . T=233 \mathrm{~K}$ and $450 \mathrm{~K}$. Measurements have been performed at zero $V B$ (line), but also for $V B<0$ (dashed lines) and $V B>0$ (dotted lines).

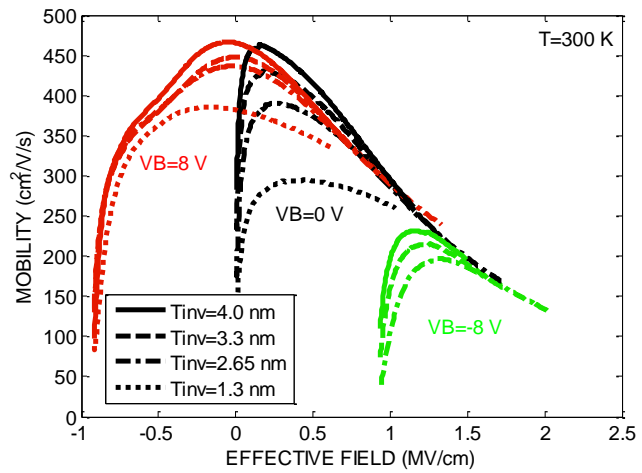

Figure 23: nFDSOI experimental effective mobility as a function of the effective field. Effective field calculated using Eq.2. ( $\eta=0.62) . T=300 \mathrm{~K}$ Devices of figure 4. 
These results confirm the difficulties to define a unique effective field in UTBB-FDSOI devices. Indeed, contrary to bulk devices, depending on gate and back voltages, carriers are not confined in a single inversion layer. The existence of multiple channels and the difference between the quality of the two interfaces (front and back) is most likely the cause of the difficulties to have a "perfect" universal trend in the whole range of effective field.

\section{CONCLUSIONS}

In this paper, we have reviewed the important aspect of the effective field and mobility in UTBB-FDSOI devices. We have demonstrated that a nearly universal trend for the mobility with respect to Eeff can be obtained only in a limited range of VB. Indeed, we showed that in forward VB conditions, a single value of $\eta$ does not make universal the mobility versus the effective field. The coexistence of multiple channels and the difference of interfaces (front and back) quality make difficult to apply the same procedure than in bulk devices for the full range of gate and back voltages.

[1] Fenouillet-Beranger, C., et al. "FDSOI devices with thin BOX and ground plane integration for $32 \mathrm{~nm}$ node and below." Solid-State Electronics 53.7 (2009): 730-734.

[2] Planes, N., et al. "28nm FDSOI technology platform for high-speed lowvoltage digital applications." VLSI Technology (VLSIT), 2012 Symposium on. IEEE, 2012.

[3] Ohata, A., et al. "Impact of back-gate biasing on effective field and mobility in ultrathin silicon-on-insulator metal-oxide-semiconductor field-effect-transistors." Journal of Applied Physics 113.14 (2013): 144514-144514.

[4] Cristoloveanu, Sorin, Noel Rodriguez, and Francisco Gamiz. "Why the universal mobility is not." Electron Devices, IEEE Transactions on 57.6 (2010): 1327-1333.

[5] D. Rideau, et al., "Mobility in High-K Metal Gate UTBB-FDSOI Devices: from NEGF to TCAD perspectives."'accepted IEDM 2013, (2013).

[6] Nier, O., et al. "Multi-scale strategy for high-k/metal-gate UTBB-FDSOI devices modeling with emphasis on back bias impact on mobility." Journal of Computational Electronics 12.4 (2013): 675-684.

[7] B.J. Sheu et P. K. Ko. A capacitance method to determine channel lengths for conventional and LDD MOSFET's. IEEE Electron Device Letters, vol. 5, 491-493, 1984

[8] Sherony, Melanie J., et al. "SOI MOSFET effective channel mobility." Electron Devices, IEEE Transactions on 41.2 (1994): 276-278.

[9] Sabnis, Anant G., and James T. Clemens. "Characterization of the electron mobility in the inverted $<100>\mathrm{Si}$ surface." Electron Devices Meeting, 1979 Internationa. Vol. 25. IEEE, 1979.

[10] Esseni, David, et al. "An experimental study of mobility enhancement in ultrathin SOI transistors operated in double-gate mode." Electron Devices, IEEE Transactions on 50.3 (2003): 802-808.

[11] Takagi, Shin-ichi, et al. "On the universality of inversion layer mobility in Si MOSFET's: Part I-effects of substrate impurity concentration." Electron Devices, IEEE Transactions on 41.12 (1994): 2357-2362.

[12] Bennamane, K., G. Ghibaudo, and A. Benfdila. "Method for extraction of $\eta$ parameter characterising $\mu$ eff against $\mathrm{E}$ eff curves in FD-SOI Si MOS devices." Electronics letters 45.12 (2009): 655-657.

[13] Van Den Daele, W., et al. "Detailed investigation of effective field, hole mobility and scattering mechanisms in GeOI and Ge pMOSFETs." Solid-State Electronics 59.1 (2011): 25-33. 
